\title{
EVALUACIÓN FORMATIVA Y COMPARTIDA EN EL MODELO INCLUSIVO RETOS INDIVIDUALES CON RESPONSABILIDAD COMPARTIDA
}

Formative and Shared Assessment in the Inclusive Model Individual Challenges with Shared Responsibility

Avaliaçao Formativa e Compartilhada no Modelo Inclusivo Desafíos Individuais com Responsabilidade Compartilhada

\section{José Manuel Castanedo Alonso (1)}

Marta Capllonch Bujosa (2)

(1) Universidad de Barcelona, España. Teléfono: +34 934035102. Correo electrónico: jcastaal7@alumnes.ub.edu

(2) Universidad de Barcelona, España. Teléfono: +34 934035102. Correo electrónico: mcapllonch@ub.edu

\section{Resumen}

Este trabajo presenta los resultados de la participación de todo el alumnado de la etapa de Educación Primaria (EP) en los procesos de evaluación en el área de Educación Física (EF) en un centro educativo público de Educación Infantil y Primaria en la Comunidad Autónoma de Cantabria durante el segundo y tercer trimestre del curso escolar 2015/2016. La evaluación formativa y compartida es un elemento clave del modelo inclusivo denominado Retos Individuales con Responsabilidad Compartida (RIRC), actualmente en proceso de validación científica. La evaluación se realiza respetando el principio de diálogo igualitario en dos momentos específicos: 1) al finalizar cada sesión, para valorar tanto el trabajo individual como el apoyo solidario a otros compañeros y compañeras para la consecución de los objetivos propuestos; 2) al finalizar cada trimestre, para establecer la calificación resultante de los acuerdos consensuados.

Palabras clave: Evaluación formativa y compartida; educación física; modelo inclusivo 


\begin{abstract}
The results of the experience with primary school students of Physical Education during the second and third term in a school in Cantabria (Sapain), school year 2015/2016, presented in this paper are about implementing assessment processes. Formative a shared assessment is one of the key elements of the inclusive model called Individual Challenges with Shared Responsibility, currently in the process of scientific validation. The assessment works in the frame of egalitarian dialogue principle in two specific times: 1) at the end of each session, to assess individual work and support to other classmates to achieve the proposed objectives; 2) at the end of each term, the assessment is done to establish the qualification resulting from consensual agreements.

Keywords: Formative and shared assessment; physical education; inclusive model

\section{Resumo}

Este trabalho apresenta os resultados da participação de todos os alunos na fase do Ensino Primário (EP) no processo da avaliação na área da Educação Física (EF) em uma escola pública da Comunidade Autônoma de Cantábria durante o segundo e o terceiro trimestre do ano letivo 2015/2016. Esta avaliação é um elemento chave do modelo inclusivo chamado Desafíos Individuais com Responsabilidade Compartilhada, atualmente em fase de validação cientifica. A avaliação, que é formativa e compartilhada, é feita após cada sessão através da igualdade de diálogo entre o grupo com o professor, para avaliar tanto o trabalho individual e a solidariedade e o apoio a outros companheiros e companheiras para atingir os objectivos propostos. Também é feito e no final de cada trimestre, para estabelecer a nota resultante de acordos consensuais.
\end{abstract}

Palavras-chave: Avaliaçao formativa e compartilhada; educação física; modelo inclusivo

\title{
1. Introducción
}

Presentamos los resultados de una experiencia de evaluación formativa y compartida (López-Pastor, 1999a, 1999b, 2000, 2004, 2006) en EF, integrada dentro del modelo inclusivo RIRC, fundamentado en evidencias científicas que persiguen la La Evaluación Formativa y Compartida en el Modelo Inclusivo Retos Individuales con Responsabilidad 
universalización del éxito educativo (Bruner, 2012; CPSE ${ }^{1}$; CREA ${ }^{2}$; Freire, 2003; Giroux, 1990; Habermas, 2001; Vygotsky, 2012, 2015) en un marco común de trabajo que supera enfoques que enfrentan aprendizaje instrumental y valores (Aubert, Flecha, García, Flecha \& Racionero, 2008). Se focaliza en la igualdad de resultados para el máximo desarrollo de todo el alumnado, transformando el contexto para dotar de diferentes niveles de apoyo según necesidades y en base a interacciones dialógicas (Oliver \& Gatt, 2010; Soler \& Flecha, 2010; Soler \& Pulido, 2012).

La evaluación formativa y compartida es clave en el modelo RIRC porque garantiza información y feedback, mejora el aprendizaje de alumnado y profesorado y respeta los principios inclusivos (Booth \& Ainscow, 2002) que sustentan el modelo: presencia, participación y aprendizaje o logro. En el proceso de diálogo y toma de decisiones conjunta que se da en la evaluación compartida, se fomenta el diálogo igualitario (Flecha, 1997), caracterizado por el respeto, la sinceridad y honestidad en los argumentos (Oliver \& Gatt, 2010; Soler \& Flecha, 2010; Soler \& Pulido, 2012) para conseguir acuerdos que mejoren la calidad educativa (Aubert et al., 2008).

\section{Contextualización}

Este trabajo forma parte de una investigación más amplia para la validación científica del modelo RIRC, que se implementó durante el segundo y tercer trimestre del curso 2015-2016 en EF en toda la etapa de EP -112 niños/as-.

\section{Diseño y desarrollo}

El modelo RIRC plantea trimestralmente variedad de retos motrices (figura 1) relacionados con los estándares de aprendizaje y vinculados a los diferentes Dominios de Acción Motriz (Larraz, 2009; López-Pastor, 2016). La figura 2 expone la distribución semanal en las sesiones de EF. Cada reto tiene una puntuación, siendo la suma de las puntuaciones de todos los retos en cada trimestre 6 puntos sobre 10. Los 4 restantes se relacionan con actitudes de respeto, esfuerzo, autonomía y responsabilidad, que denominamos puntos REAR.

1 Coalition for Psychology in Schools and Education: http://www.apa.org/ed/schools/coalition/

2 Community of Research on Excellence for All: http://crea.ub.edu/index/?lang=es

La Evaluación Formativa y Compartida en el Modelo Inclusivo Retos Individuales con Responsabilidad 


\begin{tabular}{|c|c|c|c|c|c|c|c|c|}
\hline \multicolumn{9}{|c|}{ EXPLICACION DE LOS RETOS - TRIMESTRE $3^{\circ}$} \\
\hline CURSO & COMBA & $\begin{array}{l}\text { HABILIDADES BALÓN- } \\
\text { MANO }\end{array}$ & PALAS & BÁDMINTON & MALABARES & ZANCOS & HOCKEY & BALÓN-PIE \\
\hline $1^{\circ}$ & $\begin{array}{l}\text { 1) Cruzados mantenidos } \\
\text { 2) Entrada a comba grupal } \\
\end{array}$ & $\begin{array}{l}\text { 1) Botes en equilibrio } \\
\text { mientras se pasa por un } \\
\text { banco }\end{array}$ & 1) 10 toques & & & $\begin{array}{l}\text { 1) } \\
\text { Mantenerse } \\
\text { en equilibrio } \\
y \text { andar } \$\end{array}$ & & $\begin{array}{l}\text { 1) Pases por } \\
\text { parejas con } \\
\text { pierna domi- } \\
\text { nante }\end{array}$ \\
\hline $2^{\circ}$ & $\begin{array}{l}\text { 1) Salto doble } \\
\text { 2) Entrada a comba grupal } \\
\text { con comba individual }\end{array}$ & $\begin{array}{l}\text { 1) Botes en equilibrio } \\
\text { mientras se pasa por un } \\
\text { banco } \\
\text { 2) Entrada a comba grupal y } \\
\text { botar balón }\end{array}$ & 1) 20 toques & & & $\begin{array}{l}\text { 1) } \\
\text { Mantenerse } \\
\text { en equilibrio } \\
y \text { andar } \$\end{array}$ & & $\begin{array}{l}\text { 1) Pases por } \\
\text { parejas con } \\
\text { pierna domi- } \\
\text { nante } \mathbf{4 h}\end{array}$ \\
\hline $3^{\circ}$ & $\begin{array}{l}\text { 1) Entrada a comba grupal } \\
\text { con comba individual } \\
\text { 2) Entrada a comba } \\
\text { individual }\end{array}$ & $\begin{array}{l}\text { 1) Pases sincronizados por } \\
\text { trios, al menos con } 3 \\
\text { balones }\end{array}$ & $\begin{array}{l}\text { 1) } 10 \text { toques con cada cara } \\
\text { de la pala }\end{array}$ & & $\begin{array}{l}\text { 1) Malabares } \\
\text { con } 2 \text { pelotas y } \\
\text { una mano }\end{array}$ & $\begin{array}{l}\text { 1) Andar en } \\
\text { peldaño alto } \\
\text { 1) Realizar un } \\
\text { truco }\end{array}$ & & $\begin{array}{l}\text { 1) Pases por } \\
\text { parejas con } \\
\text { pierna domi- } \\
\text { nante }\end{array}$ \\
\hline $4^{\circ}$ & $\begin{array}{l}\text { 1) } 2-2+\boldsymbol{+ 4} \\
\text { 2) Entrada y saltos en comba } \\
\text { doble grupal }\end{array}$ & $\begin{array}{l}\text { 1) Pases sincronizados por } \\
\text { trios con } 4 \text { balones } \\
\text { 2) Entrepiernas } 4 \text { manos }\end{array}$ & $\begin{array}{l}\text { 1) } 10 \text { toques con cada cara } \\
\text { de la pala y con } 2 \text { giros de } \\
\text { 360 } \\
\text { 2) Pases por parejas }\end{array}$ & & $\begin{array}{l}\text { 1) Malabares } \\
\text { con } 2 \text { pelotas y } \\
\text { una mano }\end{array}$ & & $\begin{array}{l}\text { 1) Pases por } \\
\text { parejas con } \\
\text { freestyle } \\
\text { entre pase y } \\
\text { pase }\end{array}$ & \\
\hline $5^{\circ}$ & $\begin{array}{l}\text { 1) 3-2 } \\
\text { 2) Entrada y saltos en comba } \\
\text { doble grupal }\end{array}$ & $\begin{array}{l}\text { 1) Pases sincronizados entre } \\
4 \text { personas con } 5 \text { balones } \\
\text { 2) Entrepiernas } 4 \text { manos }\end{array}$ & $\begin{array}{l}\text { 1) Pases en corto por } \\
\text { parejas } \$ \text { - } \\
\text { 2) } 3 \text { trucos freestyle }\end{array}$ & $\begin{array}{l}\text { 1) Pases en } \\
\text { parejas ith }\end{array}$ & $\begin{array}{l}\text { 1) Malabar con } \\
\text { tres pelotas } \\
\text { \$ }\end{array}$ & & $\begin{array}{l}\text { 1) Pases por } \\
\text { parejas con } \\
\text { freestyle } \\
\text { entre pase y } \\
\text { pase }+ \text { - }\end{array}$ & \\
\hline $6^{\circ}$ & $\begin{array}{l}\text { 1) } 5-2 \\
\text { 2) Salida de comba doble } \\
\text { grupal }\end{array}$ & $\begin{array}{l}\text { 1) Pases sincronizados } \\
\text { dentro de comba grupal } \\
\text { 2) Entrepiernas } 4 \text { manos }\end{array}$ & $\begin{array}{l}\text { 1) } 5 \text { trucos de freestyle } \\
\text { 2) Pases por parejas con } 2 \\
\text { pelotas }\end{array}$ & $\begin{array}{l}\text { 1) Pases en parcjas } \\
\text { 2) Pases por } \\
\text { parcjas, saltando } \\
\text { uno de la parcja en } \\
\text { comba grupal }\end{array}$ & $\begin{array}{l}\text { 1) Malabar con } \\
3 \text { pelotas ( } 2 \\
\text { vueltas) }\end{array}$ & & $\begin{array}{l}\text { 1) Pases por } \\
\text { parejas con } \\
\text { freestyle } \\
\text { entre pase y } \\
\text { pase }\end{array}$ & $\begin{array}{l}\text { 1) Dar } 10 \\
\text { toques a balón } \\
\text { con cualquier } \\
\text { parte, excepto } \\
\text { manos }\end{array}$ \\
\hline
\end{tabular}

Figura 1.

Retos de $1^{\circ}$ a $6^{\circ}$ (tercer trimestre)

\begin{tabular}{|c|c|c|c|}
\hline \multicolumn{4}{|c|}{ ORGANIZACIÓN SESIONES EF - TRIMESTRE 3ํํำ } \\
\hline & SESIÓN 1 ${ }^{\mathrm{a}}$ & SESIÓN 2a & SESIÓN 3" \\
\hline $1^{2}$ & $\begin{array}{l}10^{\prime} \text { Reto } 2 \text { comba } \\
10^{\prime} \text { Reto } 1 \text { balón-mano } \\
10^{\prime} \text { Reto } 1 \text { palas } \\
\text { Evaluación } \\
\end{array}$ & $\begin{array}{l}10^{\prime} \text { Reto } 1 \text { comba } \\
10^{\prime} \text { Reto } 1 \text { zancos } \\
10^{\prime} \text { Reto } 1 \text { balón-pie } \\
\text { Evaluación } \\
\end{array}$ & \\
\hline $2^{2}$ & $\begin{array}{l}10^{\prime} \text { Reto } 2 \text { comba } \\
10^{\prime} \text { Reto } 1 \text { balón-mano } \\
10^{\prime} \text { Reto } 1 \text { palas } \\
\text { Evaluación }\end{array}$ & $\begin{array}{l}10^{\prime} \text { Reto } 1 \text { comba } \\
10^{\prime} \text { Reto } 2 \text { balón-mano } \\
10^{\prime} \text { Reto } 1 \text { zancos } \\
10^{\prime} \text { Reto } 1 \text { balón-pie } \\
\text { Evaluación }\end{array}$ & \\
\hline $3^{2}$ & $\begin{array}{l}10^{\prime} \text { Reto } 2 \text { comba } \\
10^{\prime} \text { Reto } 1 \text { balón-mano } \\
10^{\prime} \text { Reto } 2 \text { zancos } \\
\text { Evaluación }\end{array}$ & $\begin{array}{l}10^{\prime} \text { Reto } 1 \text { comba } \\
10^{\prime} \text { Reto } 1 \text { palas } \\
10^{\prime} \text { Reto } 2 \text { balón-pie } \\
\text { Evaluación }\end{array}$ & $\begin{array}{l}10^{\prime} \text { Reto } 1 \text { comba } \\
10^{\prime} \text { Reto } 1 \text { malabares } \\
10^{\prime} \text { Reto } 1 \text { zancos } \\
\text { Evaluación }\end{array}$ \\
\hline $44^{2}$ & $\begin{array}{l}10^{\prime} \text { Reto } 1 \text { comba } \\
10^{\prime} \text { Reto } 1 \text { balón-mano } \\
10^{\prime} \text { Reto } 1 \text { palas } \\
\text { Evaluación }\end{array}$ & $\begin{array}{l}10^{\prime} \text { Reto } 2 \text { comba } \\
10^{\prime} \text { Reto } 2 \text { balón-mano } \\
10^{\prime} \text { Reto } 2 \text { palas } \\
\text { Evaluación }\end{array}$ & $\begin{array}{l}10^{\prime} \text { Reto } 2 \text { comba } \\
10^{\prime} \text { Reto } 1 \text { malabares } \\
10^{\prime} \text { Reto } 1 \text { floorball } \\
\text { Evaluación }\end{array}$ \\
\hline 5 & $\begin{array}{l}10^{\prime} \text { Reto } 1 \text { comba } \\
10^{\prime} \text { Reto } 1 \text { balón-mano } \\
10^{\prime} \text { Reto } 2 \text { palas } \\
\text { Evaluación } \\
\end{array}$ & $\begin{array}{l}10^{\prime} \text { Reto } 2 \text { comba } \\
10^{\prime} \text { Reto } 2 \text { balón-mano } \\
10^{\prime} \text { Reto } 1 \text { palas } \\
\text { Evaluación } \\
\end{array}$ & $\begin{array}{l}10^{\prime} \text { Reto } 1 \text { malabares } \\
10^{\prime} \text { Reto } 1 \text { bádminton } \\
10^{\prime} \text { Reto } 1 \text { floorball } \\
\text { Evaluación } \\
\end{array}$ \\
\hline $6^{2}$ & $\begin{array}{l}10^{\prime} \text { Reto } 1 \text { de comba } \\
10^{\prime} \text { Reto } 2 \text { de comba } \\
10^{\prime} \text { Reto } 1 \text { balón-mano } \\
10^{\prime} \text { Reto } 2 \text { balón-mano } \\
\text { Evaluación }\end{array}$ & $\begin{array}{l}10^{\prime} \text { Reto } 1 \text { de palas } \\
10^{\prime} \text { Reto } 2 \text { de palas } \\
10^{\prime} \text { Reto } 1 \text { de bádminton } \\
10^{\prime} \text { Reto } 2 \text { de bádminton } \\
\text { Evaluación }\end{array}$ & $\begin{array}{l}10^{\prime} \text { Reto } 1 \text { malabares } \\
10^{\prime} \text { Reto } 1 \text { balón-pie } \\
10^{\prime} \text { Reto } 1 \text { floorball } \\
\text { Evaluación }\end{array}$ \\
\hline
\end{tabular}

Figura 2.

Organización de retos en las diferentes sesiones de $1^{\circ}$ a $6^{\circ}$ (tercer trimestre)

La evaluación implica un trabajo continuo de guía, ayuda y mejora individual y colectiva. Consta de evaluación formativa y compartida al finalizar cada sesión para valorar el trabajo realizado en base a cuatro códigos (figura 3), y otros aspectos (agrupamientos, ayudas, conflictos, dificultades, agradecimientos...). Cualquier persona puede apoyar o rebatir las valoraciones individuales en condiciones de diálogo igualitario y argumentos de validez.

La Evaluación Formativa y Compartida en el Modelo Inclusivo Retos Individuales con Responsabilidad Compartida 


\begin{tabular}{ll}
\hline Código & Significado \\
\hline $\mathbf{0}$ & $\begin{array}{l}\text { No me he esforzado en el tiempo de trabajo, he molestado a los compañeros y compañeras } \\
\text { que estaban trabajando y/o he incumplido alguna norma (faltas de respeto) }\end{array}$ \\
\hline $\mathbf{1}$ & Me he esforzado pero no durante todo el tiempo de trabajo \\
\hline $\mathbf{2}$ & Me he esforzado durante todo el tiempo de trabajo y he mejorado en algún reto \\
\hline & Me he esforzado durante todo el tiempo de trabajo, he mejorado en algún reto y/o he sido \\
& nelidario con mis compañeros y compañeras ayudando a mejorar a quienes más lo \\
\hline
\end{tabular}

Figura 3.

Códigos de evaluación diaria

Preguntas en relación a los códigos utilizados durante la evaluación diaria (figura 4):

\begin{tabular}{ll}
\hline Código & \multicolumn{1}{c}{ Significado } \\
\hline $\mathbf{0}$ & $\begin{array}{l}\text { ¿Sabes qué norma has incumplido? ¿Eres consciente de haber faltado el respeto a alguien o } \\
\text { haberle hecho sentir mal? ¿Sabes por qué creemos que la valoración de hoy es un 0? }\end{array}$ \\
\hline $\mathbf{1}$ & ¿Qué ha pasado? ¿Por qué no has estado trabajando? Las personas que habéis trabajado con \\
& él/ella, ¿qué opináis? \\
\hline $\mathbf{3}$ & ¿Has trabajado todo el tiempo? ¿En qué has mejorado? ¿Nos lo puedes enseñar? ¿Alguien \\
& no está de acuerdo? \\
\hline & ¿A quién has ayudado? ¿Tu ayuda ha servido para que mejore? ¿En qué ha mejorado? ¿Es \\
& cierto que tu compañero/a te ha ayudado? ¿En qué has mejorado?
\end{tabular}

Figura 4.

Preguntas y códigos de evaluación

Del registro de valoraciones de cada sesión se derivan los 4 puntos REAR, donde 0 o 1 resta 0,25 puntos de los 4. Cuando alguien supera un reto, los puntos del mismo se acumulan en un banco grupal, consensuándose su reparto mediante diálogo igualitario en la evaluación formativa y compartida trimestral. Los puntos derivados de esta calificación dialogada se suman a los REAR, obteniéndose la calificación trimestral definitiva.

\section{Evaluación}

Destacamos el aumento de la conciencia del alumnado en relación a qué ha hecho, cómo y qué hacer para mejorar. La información es muy útil "porque así podemos saber La Evaluación Formativa y Compartida en el Modelo Inclusivo Retos Individuales con Responsabilidad Compartida 
todo lo que tenemos que hacer, para mejorar y todo eso”3. Al empezar con este sistema, aquellas personas que realizaban sobrevaloraciones se encontraban con argumentos válidos de compañeros/as que los cuestionaban. Así, las valoraciones pasaron a estar cargadas de sinceridad y honestidad sin mediatización de la calificación. Igualmente, las ayudas no estaban forzadas por la calificación, siendo libres y solidarias. Ayudar es importante "porque quien ayuda también aprende, además algún día tendrán que ayudarle a él”4. Los diálogos compartidos entre alumnado y maestro generan reflexiones constantes sobre la práctica que, junto al conocimiento científico acumulado, han permitido mejorar aspectos para la calidad de las clases como: libertad de interacciones para facilitar apoyos, reorganización de apoyos, ampliación del tiempo de aprendizaje, sistema de obtención de puntos...

La evaluación formativa y compartida trimestral se vive como una situación democrática, aunando participación, diálogo, negociación, respeto y sensación de justicia: “Es la primera vez que la nota que me ponen en EF es justa. Todos los años anteriores sacaba notables y sobresalientes, pero yo sabía que no lo merecía” ${ }^{5}$. La figura 5 indica los acuerdos grupales tomados para el reparto de puntos del segundo trimestre:

\begin{tabular}{ll}
\hline Curso & \multicolumn{1}{c}{ Acuerdos } \\
\hline $\mathbf{1}^{\circ}$ & Cada persona se queda con los puntos de los retos que ha superado \\
\hline $\mathbf{2}^{\circ}$ & Se reparten equitativamente los puntos conseguidos de la superación de los retos \\
\hline $\mathbf{3}^{\circ}$ & $\begin{array}{l}\text { Cada persona se queda con los puntos de los retos superados y quien quiera puede donar sus } \\
\text { propios puntos a las personas que consideren (N. le cede un punto a B; J. le cede a F., quien } \\
\text { lo agradece pero lo devuelve; C. le cede un punto a S. para que obtenga un 9) }\end{array}$ \\
\hline & $\begin{array}{l}\text { Quienes han superado todos los retos se merecen } 6 \text { puntos } \\
\text { A quienes les falta un reto por superar se quedan con 4.5 puntos }\end{array}$ \\
& $\begin{array}{l}\text { A quienes les falta más de un reto por superar se quedan con 3.75 puntos } \\
\text { Las personas que no han superado todos los retos pero que se han esforzado mucho se } \\
\text { merecen algún punto más. Esos puntos salen de las puntuaciones finales de los alumnos y } \\
\text { alumnas que deseen donar. }\end{array}$ \\
\hline $\mathbf{5}^{\circ}$ & $\begin{array}{l}\text { Cada persona se queda con los puntos de los retos que ha superado y quien quiera puede } \\
\text { donar sus propios puntos a las personas que consideren (hay muchas donaciones, pero llama }\end{array}$ \\
\hline $\mathbf{6}^{\circ}$ & $\begin{array}{l}\text { Cada persona se queda con los puntos de los retos que ha superado, teniendo la libertad para } \\
\text { donar puntos propios a compañeros/as que crea que se han esforzado o que les han ayudado }\end{array}$ \\
\hline
\end{tabular}

Figura 5.

Acuerdos de evaluación para el reparto de puntos $\left(2^{\circ}\right.$ trimestre $)$

\footnotetext{
${ }^{3}$ Alumno de $6^{\circ}$ en una entrevista grupal (segundo trimestre).

${ }^{4}$ Alumna de $2^{\circ}$ en una entrevista grupal (segundo trimestre).

${ }^{5}$ Alumno de $6^{\circ}$ en la evaluación final (segundo trimestre).

La Evaluación Formativa y Compartida en el Modelo Inclusivo Retos Individuales con Responsabilidad Compartida 
En $6^{\circ}$ se negoció una mejora del sistema de obtención de puntos, acordando conseguir puntuación extra cuando todo el grupo lograra un reto, lo que denominaron “cerrar reto”. Esta mejora se empezó a aplicar en el tercer trimestre (figura 6):

\begin{tabular}{ll}
\hline Curso & \multicolumn{1}{c}{ Acuerdos } \\
\hline $\mathbf{1}^{\circ}$ & $\begin{array}{l}\text { Cada persona se queda con los puntos de los retos que ha superado y los puntos extra se } \\
\text { reparten equitativamente }\end{array}$ \\
\hline $\mathbf{2}^{\circ}$ & Reparten de manera equitativa los puntos conseguidos de los retos y los puntos extra \\
\hline $\mathbf{3}^{\circ}$ & $\begin{array}{l}\text { Cada persona se queda con los puntos de los retos que ha superado y se reparten los puntos } \\
\text { extra conseguidos entre las personas que más se han esforzado pero no han conseguido } \\
\text { todos los retos. También pueden donar sus puntos voluntariamente. }\end{array}$ \\
\hline $\mathbf{4}^{\circ}$ & $\begin{array}{l}\text { Cada persona se queda con los puntos de los retos que ha superado y se reparten los puntos } \\
\text { extra conseguidos entre las personas más responsables y que más se han esforzado pero no } \\
\text { han conseguido todos los retos }\end{array}$ \\
\hline $\mathbf{5}^{\circ}$ & $\begin{array}{l}\text { Cada persona se queda con los puntos de los retos que ha superado y se reparten los puntos } \\
\text { extra conseguidos entre las personas que más se han esforzado pero no han conseguido } \\
\text { todos los retos }\end{array}$ \\
\hline $6^{\circ}$ & $\begin{array}{l}\text { Cada persona se queda con los puntos de los retos que ha superado y se reparten los puntos } \\
\text { extra conseguidos entre las personas que más se han esforzado pero no han conseguido } \\
\text { todos los retos }\end{array}$ \\
\hline
\end{tabular}

Figura 6.

Acuerdos de evaluación para el reparto de puntos ( $3^{\circ}$ trimestre)

El acuerdo en $2^{\circ}$ es representativo tanto de evaluación compartida como de aprendizaje compartido. Habiendo alumnado consciente de poder haber obtenido un sobresaliente con un acuerdo diferente, argumentaban: “no pasa nada, somos un grupo y hay que dividir los puntos igual entre todos. [..] quienes más hemos ayudado, hemos aprendido también”6.

\section{Conclusiones}

La evaluación formativa y compartida genera información muy valiosa que al alumnado le permite ser consciente de su aprendizaje y al maestro reflexionar sobre la práctica educativa. Esta evaluación es compatible con el aprendizaje dialógico, siendo el diálogo igualitario un principio clave para su desarrollo. Una de las futuras líneas de investigación es la participación evaluativa de familias y otro voluntariado que participa en el proceso de enseñanza-aprendizaje dentro del modelo inclusivo RIRC.

\footnotetext{
${ }^{6}$ Alumna de $2^{\text {o }}$ en la evaluación final (tercer trimestre).

La Evaluación Formativa y Compartida en el Modelo Inclusivo Retos Individuales con Responsabilidad Compartida 


\section{Referencias}

Aubert, A., Flecha, A., García, C., Flecha, R., \& Racionero, S. (2008). Aprendizaje dialógico en la sociedad de la información. Barcelona: Hipatia.

Booth, T., \& Ainscow, M. (2002). Guía para la evaluación y mejora de la educación inclusiva: desarrollando el aprendizaje y la participación en las escuelas. Madrid: Consorcio Universitario para la Educación Inclusiva. Recuperado de http://www.eenet.org.uk/resources/docs/Index\%20Castilian.pdf

Bruner, J. S. (2012). La educación, puerta de la cultura. Madrid: Antonio Machado Libros.

Flecha, R. (1997). Compartiendo palabras: El aprendizaje de las personas a través del diálogo. Barcelona: Paidós.

Freire, P. (2003). Pedagogía del oprimido (2a ed.). Madrid: Siglo XXI.

Giroux, H. (1990). Los profesores como intelectuales: Hacia una pedagogía crítica del aprendizaje. Barcelona: Paidós.

Habermas, J. (2001). Teoría de la acción comunicativa. Tomo I: Racionalidad de la acción y racionalización social. Tomo II: Crítica de la razón funcionalista (4ª ed. rev.). Madrid: Trotta.

Larraz, A. (2009). ¿Qué aprendizajes de educación física debería tener el alumnado al finalizar sexto curso de primaria. Tándem. Didáctica de la Educación Física, 29, 45-63.

López-Pastor, V. M. (Coord.) (1999a). Educación Física, Evaluación y Reforma. Segovia: Librería Diagonal.

López-Pastor, V. M. (1999b). Prácticas de evaluación en Educación Física: estudio de casos en Primaria, Secundaria y Formación del Profesorado. Valladolid: Servicio de Publicaciones de la Universidad de Valladolid.

López-Pastor, V. M. (2000). Buscando una evaluación formativa en educación física. Revista Apunts de Educación Física y Deportes, 62, 16-26.

López-Pastor, V. M. (2004). La participación del alumnado en los procesos evaluativos: la autoevaluación y la evaluación compartida en educación física. En A. Fraile La Evaluación Formativa y Compartida en el Modelo Inclusivo Retos Individuales con Responsabilidad 
Aranda, Didáctica de la Educación Física: una perspectiva crítica y transversal (pp. 265-291). Madrid: Biblioteca Nueva.

López-Pastor, V. M. (Coord.) (2006). La Evaluación en Educación Física. Revisión de los modelos tradicionales y planteamiento de una alternativa: la evaluación formativa y compartida. Retos. Nuevas tendencias en Educación física, Deporte y Recreación, 10, 31-41.

López-Pastor, V. (Coord.) (2016). Veinte años de formación permanente del profesorado, investigación-acción y programación por dominios de acción motriz. Retos, 29, 270-279. Recuperado de http://recyt.fecyt.es/index.php/retos/article/view/42494

Oliver, E., \& Gatt, S. (2010). De los actos comunicativos de poder a los actos comunicativos dialógicos en las aulas organizadas en grupos interactivos. Revista Signos, 43(Número especial), 279-294. doi: 10.4067/S0718-09342010000400002.

Soler, M., \& Flecha, R. (2010). Desde los actos de habla de Austin a los actos comunicativos: Perspectivas desde Searle, Habermas y CREA. Revista signos, 43(Supl. 2), 363-375. doi: 10.4067/S0718-09342010000400007

Soler, M., \& Pulido, C. (2012). Actos comunicativos y transformación de desigualdades sociales. Lenguaje y Textos, 36, 47-51.

Vygotsky, L. (2012). El desarrollo de los procesos psicológico superiores. Barcelona: Austral.

Vygotsky, L. (2015). Pensamiento y Lenguaje. Barcelona: Paidós.

La Evaluación Formativa y Compartida en el Modelo Inclusivo Retos Individuales con Responsabilidad 